\title{
LES investigation of two frequency effects on acoustically forced premixed flame
}

\author{
Xingsi Han ${ }^{\mathrm{a}, \mathrm{b}, *}$, Junfeng Yang ${ }^{\mathrm{c}}$, Junkui Mao ${ }^{\mathrm{a}}$ \\ ${ }^{a}$ College of Energy and Power Engineering, Nanjing University of Aeronautics and Astronautics, Nanjing 210016, PR China \\ ${ }^{b}$ Department of Aeronautics, Imperial College London, South Kensington Campus, SW7 2AZ London, UK \\ ${ }^{c}$ Department of Chemical Engineering, Imperial College London, South Kensington Campus, SW7 2AZ London, UK
}

\begin{abstract}
Turbulent lean premixed combustion has high potentials for the development of modern low $N O_{x}$ gas turbines. However, it renders the combustor prone to combustion instabilities. The non-linear response of turbulent premixed flame to external acoustic forcing plays a dominant role in the development of combustion instability. The present work describes a numerical study investigating the two frequency effects on the non-linear responses and interactions of lean premixed ethylene/air flame externally forced by strong inlet velocity oscillations. The target case is a bluff body stabilised premixed turbulent flame. Large Eddy Simulation (LES) is performed using a low-Mach number solver based on the open source CFD toolbox, OpenFOAM. The lean combustion is modelled using the Partially Stirred Reactor (PaSR) combustion model combining a reduced two-step chemical reaction mechanism. Both the unforced and forced reactive flows with single frequency forcing are simulated in order to validate the computational method. On the basis, the velocity oscillations are introduced at the inlet with two frequencies, namely the primary frequency of $f_{1}=160 \mathrm{~Hz}$ and the harmonic frequency of $f_{2}=320 \mathrm{~Hz}$. The introduction of second harmonic frequency is found to change the heat release rate fluctuation significantly. With two frequency forcing, the amplitudes of heat release responses at the primary frequency are reduced significantly, up to $70 \%$ less than those with single frequency forcing. Also the phase values are reduced/increased a lot depending on the level of second harmonic forcing. At the same time, the heat release rate fluctuations are also reduced responding at the harmonic forcing except one case where both the forcing amplitudes of the two frequencies are small. The physical mechanisms are found to be highly related to the vortex flow structures during the acoustic forcing. The central recirculation region and the side recirculation region which generates the flame shear layers in between have different responses to the acoustic forcing depending on the frequencies and amplitudes. This work confirms that LES, in this case via OpenFOAM, can be used to study the heat release responses and flame dynamics in complex cases of combustion instability with good accuracy. The study also demonstrates a possibility to design a controlling method of combustion instability by introducing a second frequency forcing.
\end{abstract}

Keywords: Lean premixed flame, Large Eddy Simulation (LES), Combustion instability, Two frequency forcing, OpenFOAM

\section{Introduction}

The gas turbine industry has been of importance for decades due to its versatile applications for power generation. Like many other methods to generate power, gas turbine engines produce harmful pollutions such as $N O_{x}$. To develop modern gas turbines, it requires high combustion performance as well as low emissions. One of the methods is to operate under lean combustion conditions, which has been drawn extensive attentions recently and appears to be quite promising $[1,2,3]$. However, a serious issue related to lean combustors is that it is more susceptible to damaging combustion instabilities [4]. Combustion instabilities generally refer to unsteady oscillations in the combustion chamber, which may inhibit the engine operation and even damage the engine components. The instabilities result from the

\footnotetext{
${ }^{*}$ Corresponding author.

Email addresses: x.han@imperial.ac.uk (Xingsi Han), junfeng.yang@imperial.ac.uk (Junfeng Yang), mjkpe@nuaa.edu.cn (Junkui Mao)
} 
coupling of the system acoustic waves and the unsteady heat release [5], which are often a consequence of multiple driving mechanisms. Recent progress in understanding combustion instability is reviewed in refs. $[1,6,7,8]$.

Due to the complex nature of combustion instability, its prediction and control at the early design stage of a gas turbine combustor is still challenging. A single model that may adequately incorporate unsteady combustion, acoustic waves, turbulence and heat transfer all together has not yet been fully developed. Alternatively, independent models are pursued that could capture some of the key mechanisms in combustion instability. One such method is the low order network combustor model that is seen as feasible and relatively successful, e.g. [5, 9, 10]. The model describes the combustor system as a network of connected modules, and the acoustic waves are very well modelled using simple analytical approaches due to the fact that the acoustic pressure is much smaller than the atmosphere pressure [4]. The coupling of the unsteady heat release to the unsteady perturbations, i.e. the response of the flame unsteady heat release rate to perturbations can be modelled via a flame model $[11,12]$.

The flame model is one of the key elements in the low order network modelling for combustion instability. This is because the flame model is the source for driving the instabilities. In the past, very simple linear flame models were used $[9,13,14,15]$ for simulating general qualitative behaviour. More recently, the flame model has been extended to the non-linear regime, known as a Flame Describing Function (FDF) [12, 16], in the form of:

$$
F\left(\omega,\left|u^{\prime}\right|\right)=\frac{Q^{\prime} / \bar{Q}}{u^{\prime} / \bar{u}}=G\left(\omega,\left|u^{\prime}\right|\right) e^{i \varphi\left(\omega,\left|u^{\prime}\right|\right)}
$$

where $Q^{\prime} / \bar{Q}$ is the normalised heat release rate fluctuation and $u^{\prime} / \bar{u}$ the normalised inlet velocity perturbation impinging on the flame. The FDF $F\left(\omega,\left|u^{\prime}\right|\right)$ is generally expressed in the frequency domain as gain (amplitude) $G\left(\omega,\left|u^{\prime}\right|\right)$ and phase $\varphi\left(\omega,\left|u^{\prime}\right|\right)$ which are functions of both forcing frequency $\omega$ and amplitude $\left|u^{\prime}\right|$.

The non-linearity of FDF is responsible for the phenomena of limit cycle oscillations [9, 17] and other non-linear effects such as instability triggering and mode switching. Several experimental studies [13, 17, 18, 19, 20] have been performed to determine the non-linear flame models for the analysis of combustion instability. Different mechanisms are explored in the experiments, such as the interactions of flame front with coherent structures [14, 17], attachment point dynamics [21], and flame quenching [18]. These studies suggest that the non-linear flame dynamics under the conditions of perturbations governs the non-linearity of FDF and finally the combustion instability.

Recently, the non-linear flame models provided by high-fidelity CFD are beginning to be exploited [22, 23, 24]. Large Eddy Simulation (LES) is capable of capturing unsteady flow and flame structure dynamics and is now used more and more to investigate turbulent combustion problems [25, 26, 27]. The investigations show that LES can give accurate prediction of flame dynamics encountered in combustion instabilities. Even more details can be explored from LES than experimental measurements due to that LES can provide more details about the full flow fields.

Nevertheless, most of the work, experimentally or numerically, on flame dynamics only considers the response of premixed flame to single frequency forcing, regarding to the combustion instability. There are few studies on flame dynamics in combustion instabilities containing more than one frequencies, such as in refs. [28, 29, 30]. Some recent studies suggest that the combustion could be coupled with the hydrodynamic flow instability [31, 32], especially when the bluff body is used as the flame holder. In this case, the vortex shedding from the flow instability gives an extra frequency besides the one from combustion instability. Moreover, an earlier experimental work [33] suggested a method to control the combustion instability by introducing a second frequency forcing. The work in refs. $[28,14]$ developed an experimental setup to study the bluff body stabilised premixed flame response to imposed inlet oscillations. Both cases are studied for external acoustic forcing with one frequency and two frequencies. It was reported that the vortex shedding has a significant impact on the flame dynamics and thus the unsteady heat release. The results also show that higher harmonics appear in some cases, even that the flame is imposed with single frequency forcing. The multiple frequencies could lead to a drastic change in the flame responses, i.e. the Flame Describing Function, and finally the combustion instability.

Motivated by those previous studies, the present work aims at LES investigation on the premixed flame response to acoustic forcing with two frequencies. The target case of the present study is the bluff-body stabilised flame studied experimentally [28, 14], where some experimental data are available for forced premixed flames. The objectives of the present paper are: (1) to perform LES studies of this turbulent fully premixed flame and to compare these with experimental data, using the open-source CFD toolbox, OpenFOAM; (2) to study the two frequency effects on the heat release responses and flame dynamics with external forcing by LES for the first time; (3) to explore the underlying 
physical mechanisms of the adding harmonic forcing effects on the flow fields compared with those forced with one single frequency. The target experimental setup is briefly described in Section 2, followed by the numerical setup for LES in Section 3. Validation of the numerical method will be presented in Section 4. Results and discussions will be given in Section 5. Conclusions are drawn in the last Section 6.

\section{Target experimental setup [14]}

The target experiment is the bluff body stabilised burner studied in ref. [14] (see Fig. 1). It has been used in earlier studies $[34,23]$. In the experiments, fully premixed reactants (ethylene and air with an equivalence ratio of $\phi=0.55$ ) are supplied through the plenum and the flame is stabilised by a bluff body. In the present LES, only part of the combustor is used for the simulations as shown in Fig. 1. The inlet pipe has an inner diameter of $35 \mathrm{~mm}$ and outer diameter of $8 \mathrm{~mm}$, and the conical bluff body has a diameter of $d=25 \mathrm{~mm}$. A quartz cylinder is used to confine the flame, with the inner diameter of $70 \mathrm{~mm}$ and length of $80 \mathrm{~mm}$. The bulk velocity $V_{b}$ at the combustor inlet is $V_{b}=9.9$ $\mathrm{m} / \mathrm{s}$ and it is $V_{0}=5.17 \mathrm{~m} / \mathrm{s}$ at the computational inlet (see Fig. 1).
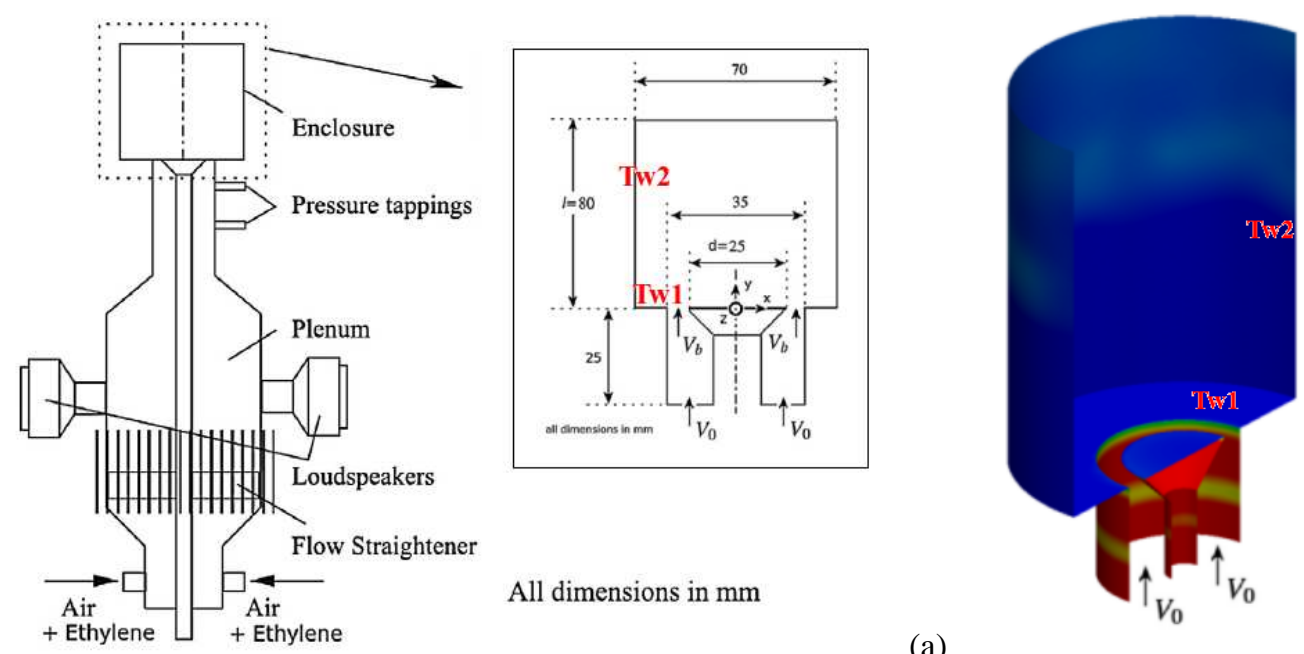

(a)

Figure 1: (a) Schematic of the experimental setup [14], with a cut $(z=0)$ for the computational domain; (b) half of the whole computational domain and the solid walls with heat loss are marked with $T_{w 1}$ and $T_{w 2}$, respectively.

In the experiments, two loudspeakers are employed to generate the acoustic forcing which introduces velocity oscillations at the plenum before entering the combustor. For premixed flame, the equivalence ratio is constant and the heat release rate fluctuations are dominated by velocity fluctuations upstream of the flame. Both single frequency forcing and two frequency forcing are conducted in the experiments. The heat release rate was measured with $\mathrm{OH}^{*}$ and $\mathrm{CH}^{*}$ chemiluminescence. It was also possible to be obtained from the phase-averaged FSD (Flame Surface Density) images via PLIF (Planar Laser-Induced Fluorescence).

\section{Numerical methods for LES}

Large Eddy Simulation (LES) is widely recognised as an accurate method for reactive flow simulations $[2,35]$ and recently it has been exploited in the context of combustion instability [23,36]. To study the two frequency effects, the open source CFD toolbox, OpenFOAM is employed in the present study, with a modified version of the reactingFOAM solver - this has been applied in previous LES studies of partially-premixed flame [23].

For reactive flow with large density fluctuations, the governing equations are the Favre-filtered Navier-Stokes equations of mass, momentum, species mass fraction and energy. The continuity and momentum equations are:

$$
\frac{\partial \bar{\rho}}{\partial t}+\frac{\partial \bar{\rho} \widetilde{U}_{i}}{\partial x_{i}}=0
$$


Table 1: The two-step $\mathrm{C}_{2} \mathrm{H}_{4} /$ Air chemical reaction mechanism [42].

\begin{tabular}{lccc}
\hline Reaction & $A\left(\mathrm{~cm}^{3} / \mathrm{mol} \mathrm{s}\right)$ & $\beta$ & $E_{a}(\mathrm{cal} / \mathrm{mol})$ \\
\hline $\mathrm{C}_{2} \mathrm{H}_{4}^{0.50}+2 \mathrm{O}_{2}^{0.65}=>2 \mathrm{CO}+2 \mathrm{H}_{2} \mathrm{O}$ & $2.0 \times 10^{10}$ & 0.00 & $3.55 \times 10^{4}$ \\
$\mathrm{CO}+0.5 \mathrm{O}_{2}<=>\mathrm{CO}_{2}$ & $2.0 \times 10^{8}$ & 0.80 & $1.20 \times 10^{4}$ \\
\hline
\end{tabular}

$$
\frac{\partial \bar{\rho} \widetilde{U}_{i}}{\partial t}+\frac{\partial \bar{\rho} \widetilde{U}_{i} \widetilde{U}_{j}}{\partial x_{j}}=-\frac{\partial \bar{p}}{\partial x_{i}}+\frac{\partial}{\partial x_{j}}\left(\bar{\mu}\left[\frac{\partial \widetilde{U}_{i}}{\partial x_{j}}+\frac{\partial \widetilde{U}_{j}}{\partial x_{i}}\right]\right)-\frac{\partial \tau_{i j}}{\partial x_{j}}
$$

where $\bar{\mu}$ is the laminar viscosity and modelled by Sutherland's law. The subgrid scale stress tensor $\tau_{i j}=\bar{\rho}\left(\widetilde{U_{i} U_{j}}-\right.$ $\left.\widetilde{U}_{i} \widetilde{U}_{j}\right)$ is determined by the popular Smagorinsky subgrid scale model [37], with the turbulent viscosity calculated by:

$$
\mu_{s g s}=\bar{\rho}\left(C_{s} \Delta\right)^{2}|\widetilde{S}|
$$

where the model constant $C_{s}$ is equal to $0.167,|\widetilde{S}|$ is the strain rate magnitude of the resolved velocity defined as $|\widetilde{S}|=\sqrt{2 \widetilde{S}_{i j} \widetilde{S}_{i j}}$, and $\Delta$ is the filter cutoff width. Note that the symbol ${ }^{-}$denotes the spatial filtering used in the LES and the symbol denotes density-weighted filtering, defined as $\widetilde{\psi}=\overline{\rho \psi} / \bar{\rho}$ for an arbitrary variable $\psi$.

For the Smagorinsky LES model, it is found that the modelled turbulent viscosity, $\mu_{s g s}$, is generally too high in the near wall regions [38]. The turbulent viscosity (Eq.(4)) should be damped via the model for van Driest damping. In OpenFOAM, the damping is derived by changing the filter width, $\Delta$, in the form of:

$$
\Delta=\min \left(\Delta_{m},\left(\frac{\kappa}{C_{\Delta}}\right) y_{w}\left(1-e^{-y^{+} / A^{+}}\right)\right)
$$

where $\Delta_{m}$ is the cubic root of the cell volume, $\kappa=0.4187$ is the von Karman constant, $C_{\Delta}=0.158, A^{+}=26, y_{w}$ represents the distance to the wall, and $y^{+}$describes the dimensionless distance to the wall calculated from the wall shear stress.

The filtered equations for the mass fractions of chemical species and energy contain subgrid fluxes and filtered chemical source terms, which are unknown and need models to be closed. Following ref. [4], the gas mixture is presumed to be ideal, linearly viscous, with Fourier heat conduction and Fickian diffusion. As the energy equation is solved, heat loss effects can be considered. For the target case, the air and fuel are fully premixed prior to the combustor. The turbulence and combustion interactions are modelled by the PaSR (Partially Stirred Reactor) model $[39,40]$ in the present LES study.

In the PaSR approach of modelling the filtered reaction source term $\overline{\dot{\omega}_{j}}$, it is assumed that the flow in a computational cell can be split into two parts; the fine structures in which mixing and reactions are assumed to take place, and the surroundings dominated by the large scale structures. The relative sizes of the two parts in the cell are governed by the combustion time and turbulent mixing time. The reaction rate for $i$-th species can then be calculated by the reactive volume fraction, $\kappa$, as proposed in ref. [41]:

$$
\frac{\partial C^{i}}{\partial t}=\frac{C_{1}^{i}-C_{0}^{i}}{\Delta t}=\kappa R R_{i}\left(C_{1}^{i}\right)
$$

where the term $R R_{i}$ is the laminar Arrhenius reaction source term, i.e. $R R_{i}=\dot{\omega}_{i}\left(\bar{\rho}, \widetilde{T}, \widetilde{Y}_{j}\right)$. Eventually, the turbulencecombustion coupling in PaSR model is reduced to the modelling of reactive volume fraction, $\kappa$, which can be modelled as [41]:

$$
\kappa=\frac{\tau_{c}}{\tau_{c}+\tau_{m}}
$$

where $\tau_{m}$ is the turbulent mixing time scale and $\tau_{c}$ the reaction time scale.

To determine the reaction time scale $\tau_{c}$, a reduced two-step six-species global chemical mechanisms of ethylene/air is applied [42], with the Arrhenius law parameters shown in Table 1. For the lean premixed flame of ethylene/air 
$(\phi<1.2)$, the reduced mechanism can correctly reproduce the laminar flame speed, and thus suitable for the present study where $\phi=0.55$. Computation costs can be significantly reduced by the reduced mechanism compared to the comprehensive ones. Another important parameter in the PaSR model is the turbulent mixing time, $\tau_{m}$ (see Eq. (7)). Here, it is modelled based on two time scales: the subgrid velocity stretch time and Kolmogorov time, in the form of $[43,44]$ :

$$
\tau_{m}=c_{m} \sqrt{\tau_{\Delta} \tau_{K}}
$$

where the subgrid time scale $\tau_{\Delta}$ and Kolmogorov time scale are calculated by:

$$
\tau_{\Delta}=\frac{\Delta}{u^{\prime}}=\frac{\Delta}{\sqrt{2 k / 3}} ; \quad \tau_{K}=\left(\frac{v}{\varepsilon}\right)^{1 / 2}
$$

with $\Delta$ the computational cell scale, $k$ the subgrid turbulent kinetic energy, $\varepsilon$ the subgrid dissipation rate and $v$ the laminar kinematic viscosity. Note that the model constant $c_{m}$ in Eq.(8) depends on specific flow configurations, and a value of 0.6 is used for the present LES based on tests of simulation experiments.

The open source CFD toolbox, OpenFOAM (version 2.3.0) is applied with a modified low-Mach number reactingFOAM solver and the new turbulent mixing time model (Eq.(8)) is incorporated in the code. The C++ library OpenFOAM has been previously used for a wide range of reactive flow simulations. It is based on a second order accurate finite volume method and the pressure-velocity is coupled through PIMPLE algorithm. All spatial derivatives are approximated by second order central difference scheme and the second order implicit Crank-Nicolson scheme is used for time discretisation. The computational domain is shown in Fig. 1 including the coordinate system. An unstructured mesh is applied for the present LES, with a final mesh containing about 2.48 million cells after several simulation tests.
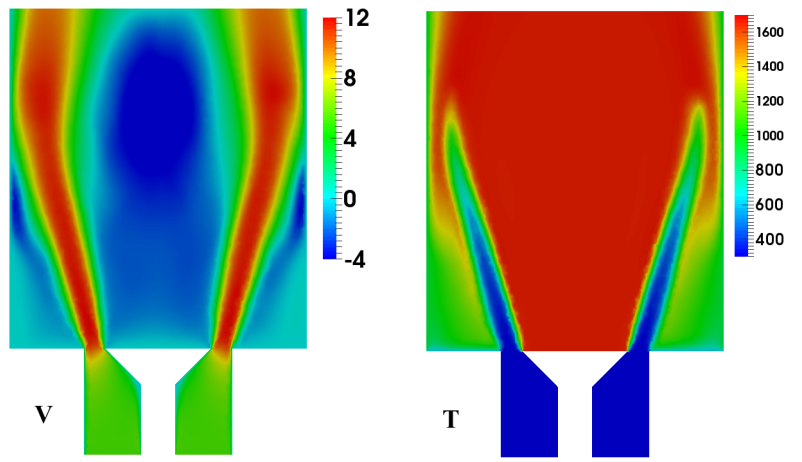

Figure 2: Time-averaged flow fields of the unforced reactive flow from the present LES: the axial velocity $V(\mathrm{~m} / \mathrm{s})($ left) and the temperature $T$ (K) (right), at a $z$-cut of $z=0$.

The main objective of the present work is to study the two frequency effects of the acoustic forcing on the unsteady heat release response for the premixed flame. To emulate the acoustic forcing, the inlet velocity is superimposed with oscillations, based on previous studies $[23,24]$, with the form:

$$
V=V_{0}\left[1+A_{1} \sin \left(2 \pi f_{1} t\right)+A_{2} \sin \left(2 \pi f_{2} t\right)\right]+V_{\text {noise }}
$$

where $A_{1}$ is the primary normalised velocity forcing amplitude and $f_{1}$ the primary forcing frequency, and $A_{2}$ is the harmonic forcing amplitude and $f_{2}$ the harmonic forcing frequency. Consistent to the experiments [14], the first primary forcing frequency is $f_{1}=160 \mathrm{~Hz}$ and the second harmonic forcing frequency is $f_{2}=320 \mathrm{~Hz}$. These two frequencies can be considered as the fundamental and first higher harmonic (twice of the fundamental) frequency combination. It should be noted that $A_{1}$ and $A_{2}$ cannot be independently specified in the experiments [28, 14]. The explanation is given as following. The output forcing signals in the experiments are generated by two loudspeakers via adjusting the peak-to-peak voltage of the input signals. When forcing with only single primary frequency of $f_{1}=160$ $\mathrm{Hz}$, the forcing energy can be generated/distributed into higher harmonics, i.e. $f_{2}=320 \mathrm{~Hz}$, which means that $A_{1}$ and 
$A_{2}$ are coupled with each other in the experiments due to the coupling of all acoustic waves in the combustor system. However, in the present simulations, the values of $A_{1}$ and $A_{2}$ can be varied independently and the effects of harmonic part can be explored more accurately than those from the experimental measurements. This also demonstrates the advantages of applying numerical methods to study the acoustic forcing problem with two frequency co-existing.

All boundaries other than the inlet and outlet are treated as solid walls, where non-slip wall conditions are applied. Thermal effects at the wall, i.e. heat loss, can modify the flame dynamics and heat release response, which have been observed in the experiments $[45,46]$. To account for the heat loss through the walls (see Fig. 1), a lower temperature than the adiabatic temperature is imposed on those walls, i.e. $T_{w 1}=600 \mathrm{~K}$ and $T_{w 2}=900 \mathrm{~K}$, which are specified based on previous studies [23,47]. Adiabatic conditions are applied for all other walls.

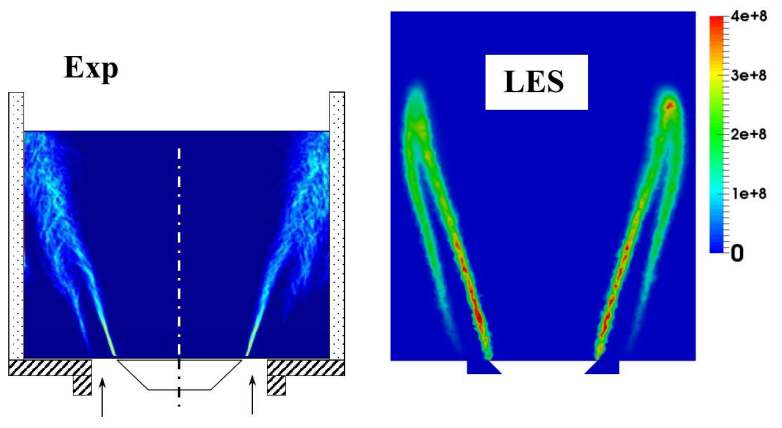

Figure 3: Comparison of the time-averaged flow fields for the unforced reactive flow: the FSD image from experiments [14] (left) and the volumetric heat release rate in $\mathrm{W} / \mathrm{m}^{3}$ from the present LES (right).

\section{Validation of the LES method}

To validate the numerical method, LES is firstly performed for the unforced reactive flow. Figure 2 shows the time-averaged flow fields of axial velocity and temperature for the unforced reactive flow. It demonstrates that a "M"shaped flame is formed along the shear layers of the jet and stabilised by the bluff body. A central recirculation region after the bluff body and a side recirculation region along the side wall can be clearly observed. The temperature flow field shows the flame fronts of the premixed flame. The heat release rate results from the present LES are compared with the FSD image from experimental measurements [14] in Fig. 3. The agreement between the prediction and experiment is good. It should be noted that due to the heat loss through the walls, the temperature is lower than the adiabatic temperature in the side recirculation region (see Fig. 2) and thus the outer branches of the flame fronts are weaker than those inner ones (see Fig. 3). Previous LES study [22] of the unforced reactive case applies a FSD combustion model which does not solve the energy equation and thus cannot account for the heat loss. The previous LES prediction [22] is thus less well than the present LES prediction on the flames.

The forced reactive cases are also performed to validate the present LES method. To the end, the inlet velocity is imposed with fluctuations following the form as shown in Eq.(10). It could be expected that the flame and thus the heat release will oscillate with the inlet velocity oscillations. The heat release signals integrated over the whole computational domain are recorded during the simulations and the time series are then transformed to the frequency domain using a Fourier Transform technique. The gain and phase of the FDF can be obtained from its definition in Eq.(1). For the validation purpose, only single frequency forcing is performed in this section, which means that there is no forcing of $f_{2}=320 \mathrm{~Hz}$ (i.e. $A_{2}=0$ ) when forcing at $f_{1}=160 \mathrm{~Hz}$, and $A_{1}=0$ when forcing at $f_{2}=320 \mathrm{~Hz}$, using the inlet velocity form shown in Eq.(10).

For only single frequency forcing at $f_{1}=160 \mathrm{~Hz}$, the normalised amplitude of heat release rate fluctuation as a function of forcing amplitude $A_{1}$ is shown in Fig. 4 with comparisons to experiments [14] and previous LES results [22]. It can be observed that the present LES results agree well with the experimental measurements from $\mathrm{OH}^{*}$ and $\mathrm{CH}^{*}$ chemiluminescence, and even better than previous LES predictions. This implies that the heat loss has considerable impacts on the heat release results and should be accounted for in the simulations. The results show that 
the heat release response is linear up to around $A_{1}=0.15$ and then becomes non-linear until it saturates at around $A_{1}=0.6$. The phase difference of the heat release fluctuation from the inlet velocity fluctuation is also captured well by the present LES, as shown in Fig. 4(b). When forcing with a single frequency of $f_{2}=320 \mathrm{~Hz}$, the heat release responses differently as shown in Fig. 5. The amplitude of heat release fluctuation is much smaller than that at the lower frequency of $f_{1}=160 \mathrm{~Hz}$ and the phase increases significantly with increasing the forcing amplitude $A_{2}$. Nevertheless, the present LES predictions match well with the experiments and the previous LES results overall.
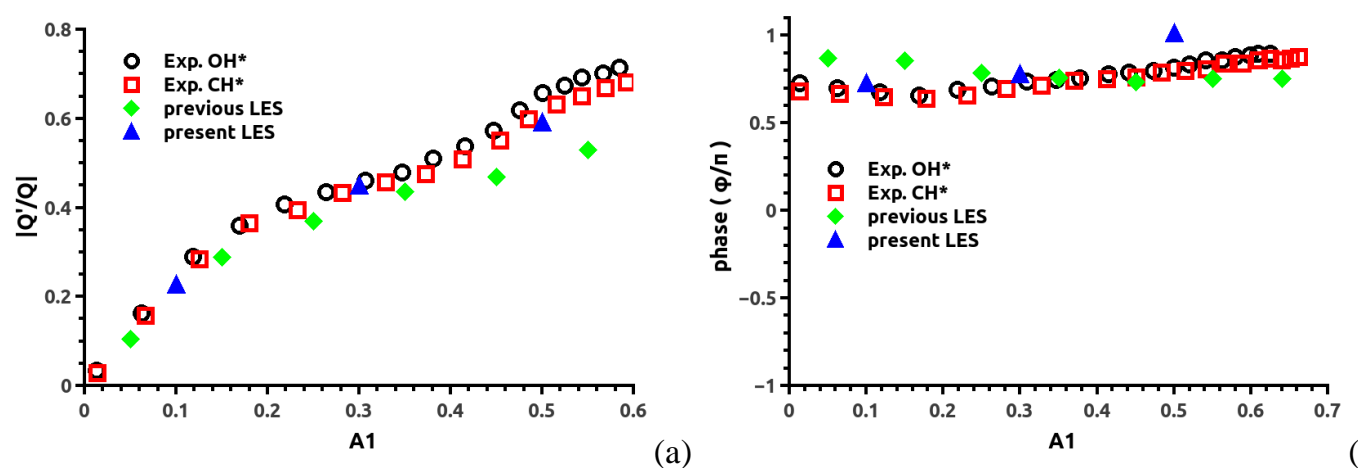

Figure 4: (a) Dependence of the amplitude of the heat release rate response with velocity fluctuation amplitude $A_{1}$; (b) the dependence of the phase of the heat release rate response $\varphi$ (Eq. (1)), at single forcing frequency $f_{1}=160 \mathrm{~Hz}$. Experimental data are from [14] and previous LES refers to results from [22].
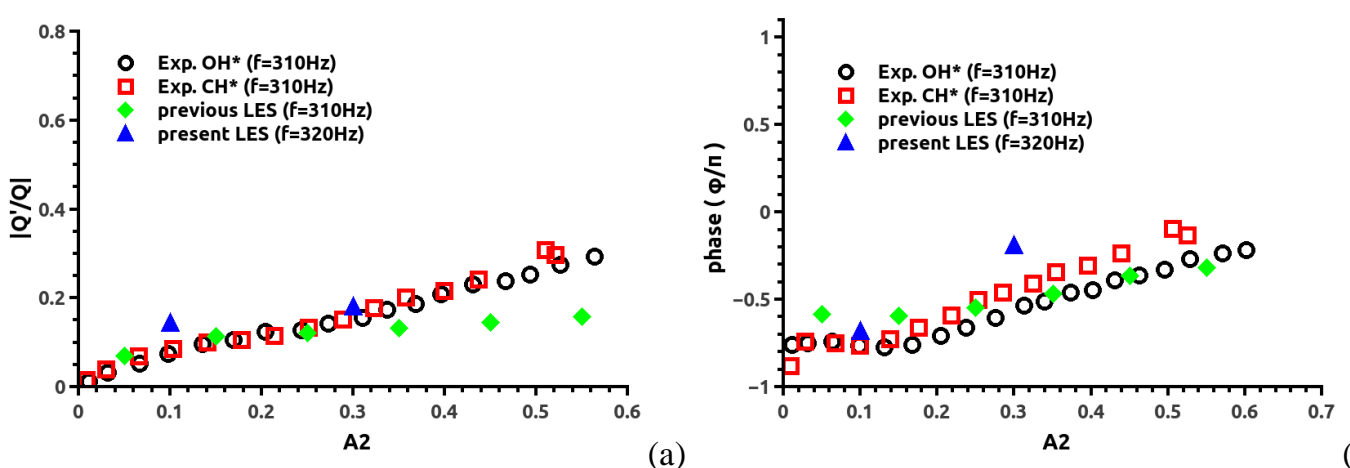

Figure 5: (a) Dependence of the amplitude of the heat release rate response with velocity fluctuation amplitude $A_{2}$; (b) the dependence of the phase of the heat release rate response $\varphi$ (Eq. (1)), at single forcing frequency $f_{2}=320 \mathrm{~Hz}$. Experimental data are from [14] and previous LES refers to results from [22].

To further validate the present LES method, unsteady flame dynamics with single frequency forcing are also examined and compared with the available experiments $[14,15]$, as shown in Fig. 6. It clearly demonstrates the deformation of the flame base, later resulting in a mushroom-shaped flame front - similar to previous results [22] with a forcing of $f_{1}=160 \mathrm{~Hz}$ and $A_{1}=0.64$. The present LES captures the flame evolution quite well compared with experiments at all the phase angles. Note that the mushroom-shaped vortex is the main factor for the non-linear heat release response behaviour which has been discussed previously [22].

To summarise, the present LES method resolves the reactive flow fields and unsteady heat release response quite well compared to available experimental data, for both cases without or with acoustic forcing.

\section{Results and discussions: two frequency effects}

The previous section confirms that the present LES method based on OpenFOAM toolbox can capture the reactive flow well with external acoustic forcing. The numerical method can thus be used for investigation of two frequency effects on the forced premixed flame. 
Table 2: The simulation cases with two frequency forcing. $A_{1}$ and $A_{2}$ are the forcing amplitudes corresponding to the two forcing frequencies of $f_{1}=160 \mathrm{~Hz}$ and $f_{2}=320 \mathrm{~Hz}$, respectively.

\begin{tabular}{ccccc}
\hline \multirow{2}{*}{$A_{2}$} & \multicolumn{4}{c}{$A_{1}$} \\
\cline { 2 - 5 } & 0.1 & 0.2 & 0.3 & 0.4 \\
\hline 0.1 & $\mathrm{C} 11$ & $\mathrm{C} 21$ & $\mathrm{C} 31$ & $\mathrm{C} 41$ \\
0.3 & $\mathrm{C} 13$ & $\mathrm{C} 23$ & $\mathrm{C} 33$ & $\mathrm{C} 43$ \\
\hline
\end{tabular}

Table 3: Pair of the maxima and minima in each cycle for the incoming velocity signal shown in Eq.(10) with two frequency forcing for different simulation cases. PI means one pair and PII means two pairs.

\begin{tabular}{ccccc}
\hline \multirow{2}{*}{$A_{2}$} & \multicolumn{4}{c}{$A_{1}$} \\
\cline { 2 - 5 } & 0.1 & 0.2 & 0.3 & 0.4 \\
\hline 0.1 & PII & PI & PI & PI \\
0.3 & PII & PII & PII & PII \\
\hline
\end{tabular}

The inlet velocity is imposed with oscillations following the form shown in Eq.(10), where $f_{1}=160 \mathrm{~Hz}$ is the primary forcing frequency and $f_{2}=320 \mathrm{~Hz}$ the added harmonic forcing frequency. For the primary frequency of $f_{1}=160 \mathrm{~Hz}$, four forcing amplitudes are performed, i.e. $A_{1}=0.1,0.2,0.3$ and 0.4 , and two forcing amplitudes are applied for the harmonic forcing frequency of $f_{2}=320 \mathrm{~Hz}$, i.e $A_{2}=0.1$ and 0.3 . The combinations of the two forcing frequencies are summarised in Table 2 and shows the simulation cases to study the two frequency effects in this section.
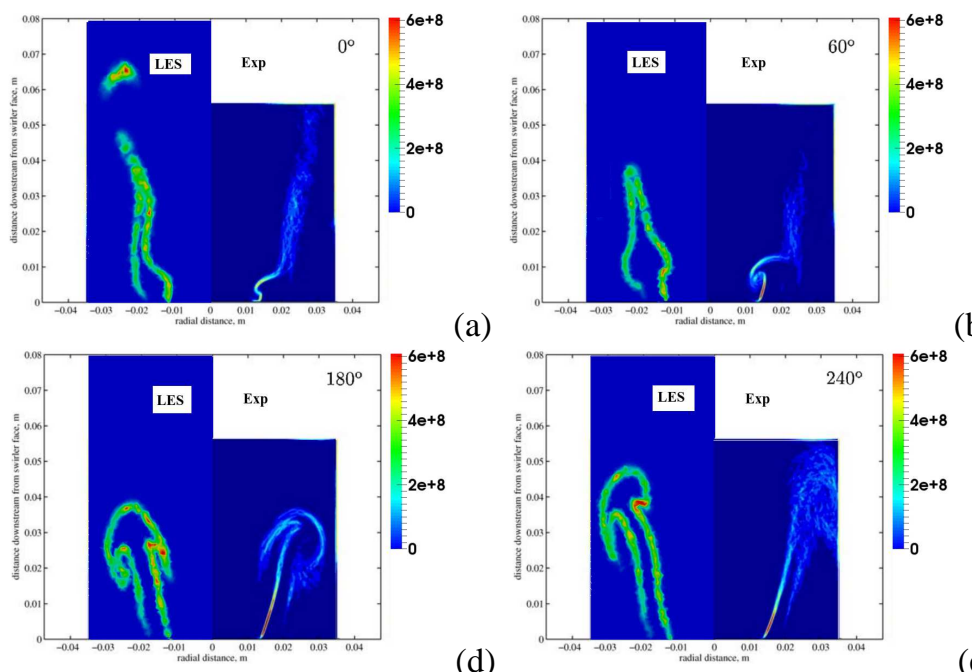

(d)

(e)

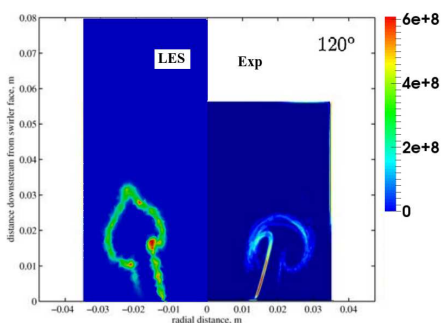

(b)

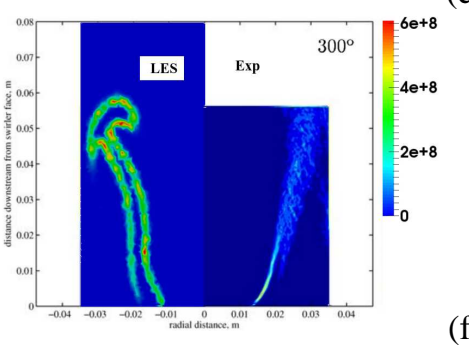

(c)

(f)

Figure 6: Comparison of the heat release rate (in $\mathrm{W} / \mathrm{m}^{3}$ ) from the present LES (left) and the FSD image from the experiments [14, 15] (right) at different phase angles with single frequency strong forcing of $f_{1}=160 \mathrm{~Hz}$ and $A_{1}=0.5$.

For the inlet velocity signals following the form shown in Eq.(10), there could be two pairs of maxima and minima in each forcing cycle due to having two frequencies. From theoretical analysis as detailed in ref. [28], there are two pairs when $A_{2} / A_{1}>0.5$ and only one pair otherwise. Based on this conclusion, all the simulation cases shown in Table 2 could be summarised in Table 3 regarding to how many pairs of maxima and minima in one forcing cycle. One example for the cases C41 and C43 is given in Fig. 7 where both the inlet velocity and unsteady heat release rate signals are shown from the present LES. It can be seen that case C43 has two pairs, while C41 only one pair for 


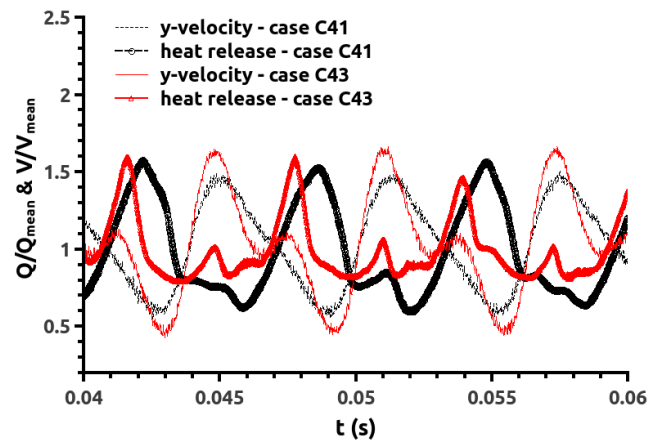

Figure 7: Examples of the normalised inlet velocity (in $y$ direction) and corresponding heat release rate signals at different forcing conditions: cases C41 and C43, from the present LES.

the inlet velocity signal. However, the heat release rate signals show a more complex pattern than the inlet velocity signals, and two pairs can be observed for both the two cases in one forcing cycle. This implies that the heat release rate could response at both of the two frequencies of $f_{1}=160 \mathrm{~Hz}$ and $f_{2}=320 \mathrm{~Hz}$ even there is only a small number of harmonic forcing added in the inlet velocity.
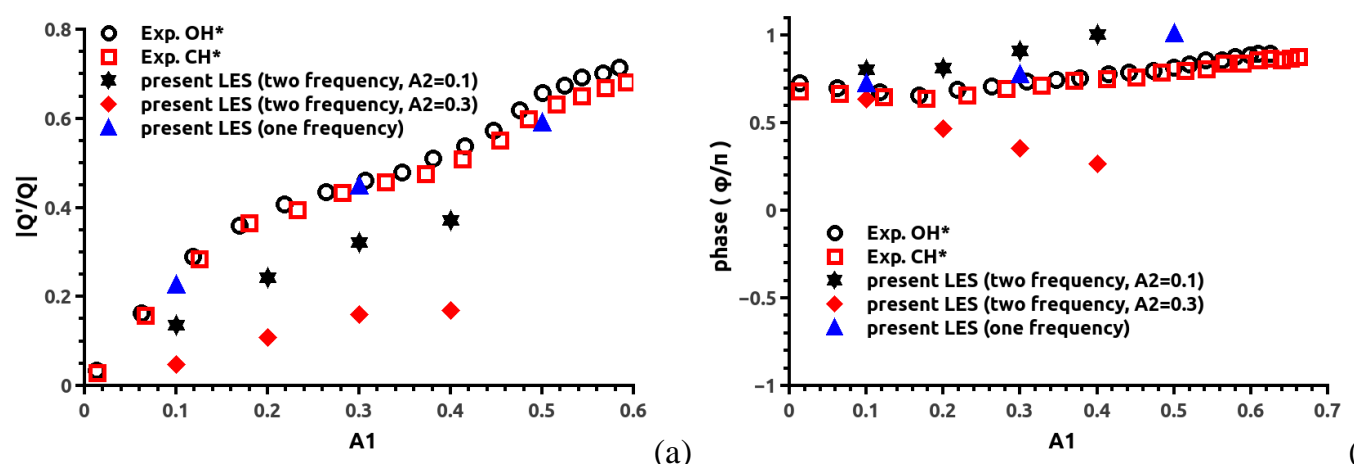

(a)

Figure 8: (a) Dependence of the amplitude of the heat release rate response with velocity fluctuation amplitude $A_{1}$; (b) the dependence of the phase of the heat release rate response $\varphi$ (Eq. (1)), at two frequency forcing with different forcing amplitudes $A_{1}$. Focus on the heat release response at the primary forcing frequency $f_{1}=160 \mathrm{~Hz}$. Experimental data are from [14] for single frequency forcing at $f_{1}=160 \mathrm{~Hz}$.
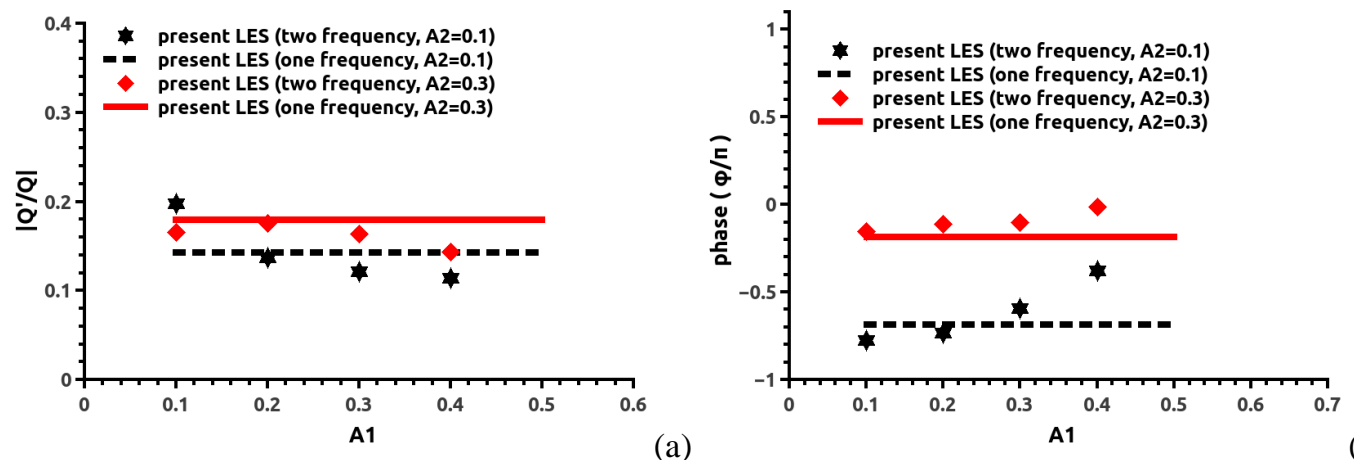

(a)

Figure 9: (a) Dependence of the amplitude of the heat release rate response with velocity fluctuation amplitude $A_{1}$; (b) the dependence of the phase of the heat release rate response $\varphi$ (Eq. (1)), at two frequency forcing with different forcing amplitudes $A_{1}$. Focus on the heat release response at the harmonic forcing frequency $f_{2}=320 \mathrm{~Hz}$.

Fourier Transforms are performed to process the time series of the heat release rate and inlet velocity. For two 
frequency forcing, the present LES results demonstrate that all the cases shown in Table 2 produce a unsteady heat release rate that responses at both of the two forcing frequencies at the same time. Firstly, for the primary forcing at $f_{1}=160 \mathrm{~Hz}$, the effects of adding harmonic forcing of $f_{2}=320 \mathrm{~Hz}$ on the heat release rate are examined and the results are shown in Fig. 8 where only the responses at $f_{1}=160 \mathrm{~Hz}$ are presented. It shows that the normalised amplitudes of heat release rate fluctuation at $f_{1}=160 \mathrm{~Hz}$ are reduced compared with those with single forcing of $f_{1}=160 \mathrm{~Hz}$. The more adding of harmonic forcing of $f_{2}=320 \mathrm{~Hz}$, the smaller of the amplitude of heat release response at $f_{1}=160 \mathrm{~Hz}$. The amplitude of the response is reduced up to $70 \%$ from the single frequency forcing conditions. Moreover, it seems that the introduction of secondary harmonic forcing changes the dependence of response from highly non-linear (with single frequency forcing) to be more linear. The observation is consistent with the experiments $[14,28]$. Note that due to the highly coupling of forcing amplitudes of $A_{1}$ and $A_{2}$ in the experiments $[14,28]$, the experimental data cannot be used here for the quantitative comparisons with the present LES prediction. For the phase results shown in Fig. 8(b), the effects of harmonic forcing behave differently. With a relatively small harmonic forcing amplitude, i.e. $A_{2}=0.1$, the phase results are slightly increased compared with single frequency forcing of $f_{1}=160 \mathrm{~Hz}$, while the tendency of phase is changed completed when the harmonic forcing is relatively large, such as $A_{2}=0.3$ that the phase is decreasing with increasing $A_{1}$. The tendency also agrees with the experimental observations $[14,28]$.
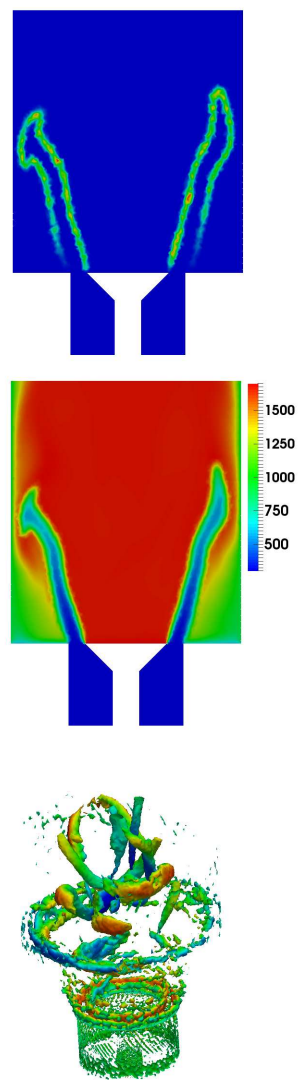

(a)
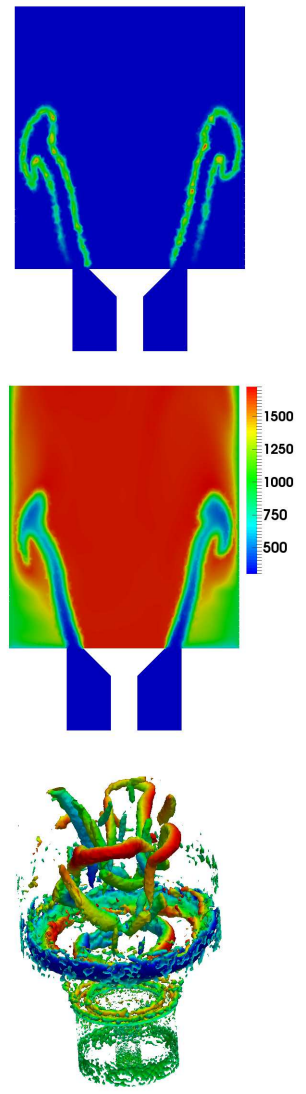

(b)
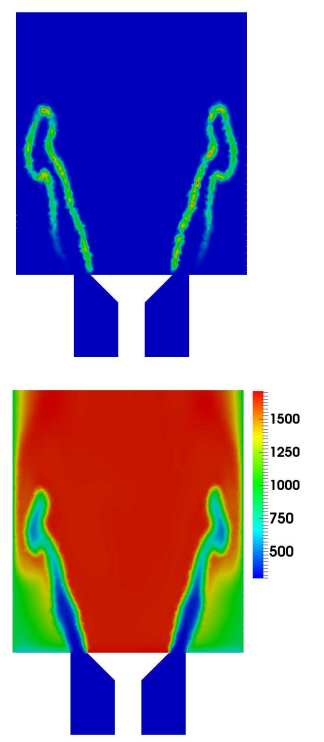

(c)

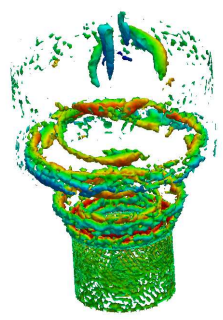

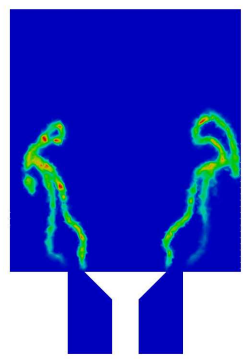
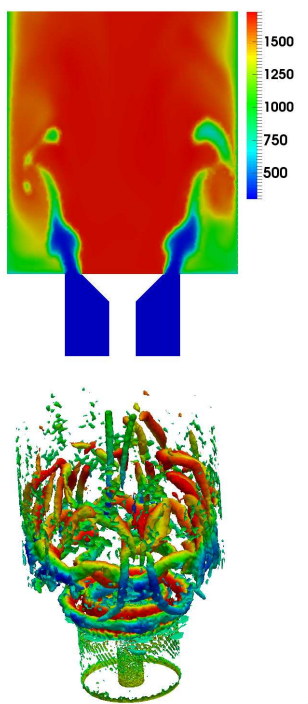

(d)

Figure 10: Flow fields with the single frequency forcing for different cases from the present LES: (a) $f_{1}=160 \mathrm{~Hz}$ and $A_{1}=0.1$; $(\mathrm{b}) f_{1}=160$ $\mathrm{Hz}$ and $A_{1}=0.3$; (c) $f_{2}=320 \mathrm{~Hz}$ and $A_{2}=0.1$; (d) $f_{2}=320 \mathrm{~Hz}$ and $A_{2}=0.3$, showing the volumetric heat release rate (top), temperature (K) (middle) and iso-surface of the second invariant of the velocity gradient $\left(Q=1.0 \times 10^{6} \mathrm{~s}^{-2}\right)$ coloured by the axial velocity (bottom).

As for the heat release response at the harmonic forcing of $f_{2}=320 \mathrm{~Hz}$ under two frequency forcing conditions, the results are given in Fig. 9. It can be seen that the amplitudes of heat release rate fluctuation at $f_{2}=320 \mathrm{~Hz}$ are generally decreased compared with the single frequency forcing of $f_{2}=320 \mathrm{~Hz}$, which means that the heat release fluctuations at both of the forcing frequencies are depressed when the harmonic forcing is introduced, implying that 
the whole combustion system becomes more stable. However, there is an exception, i.e. the case C11, in which the heat release amplitude is increased at $f_{2}=320 \mathrm{~Hz}$ as well as decreased at $f_{1}=160 \mathrm{~Hz}$. It implies that the forcing energy is converted from the primary forcing to the harmonic forcing in the case $\mathrm{C} 11$. All the other cases have complex interactions and both of the forcing responses are suppressed at the same time. The phase results behave similarly and their values are increased by the influence of primary forcing, except for the cases $\mathrm{C} 11$ and $\mathrm{C} 21$. The case of harmonic forcing with $A_{2}=0.1$ is influenced differently by the primary forcing regarding to the level of forcing amplitude $A_{1}$, i.e. there is a turning point around $A_{1}=0.2$. While for harmonic forcing with $A_{2}=0.3$, the influence of the primary forcing has the same tendency, i.e. the amplitudes are decreased and phases are increased.
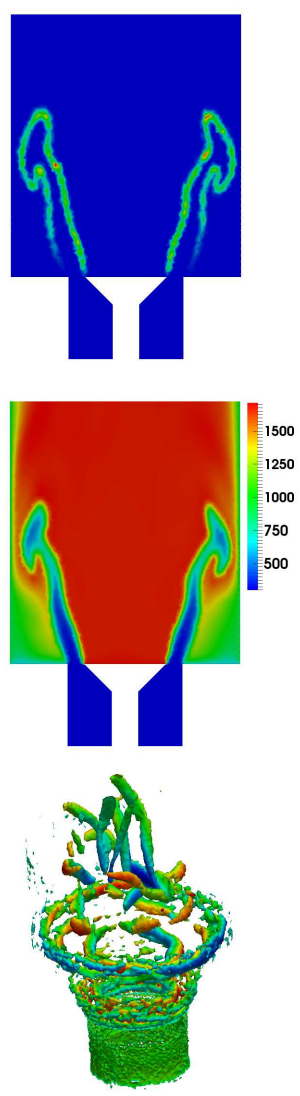
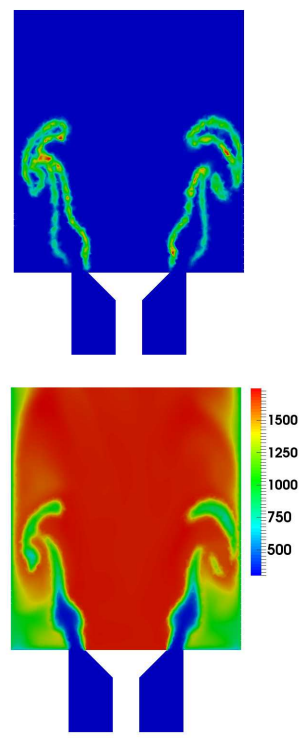

(a)

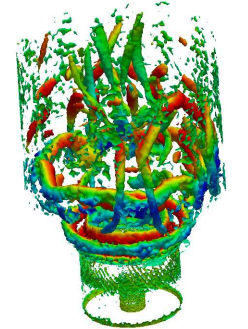

(b)
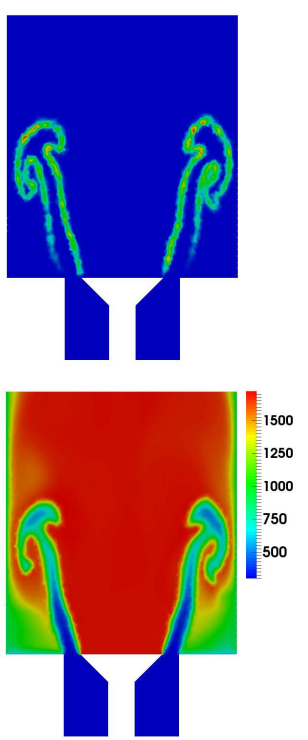

(c)

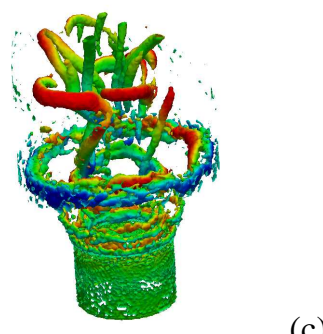

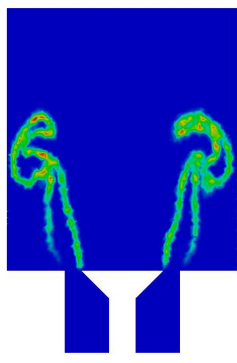
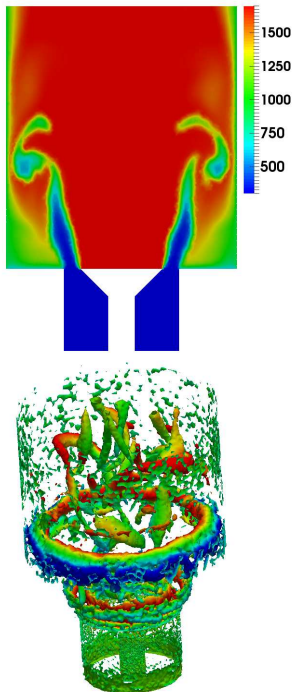

(d)

Figure 11: Flow fields with two frequency forcing for different cases from the present LES: (a) case C11; (b) case C13; (c) case C31; (d) case C33 (see Table 2), showing the volumetric heat release rate (top), temperature (K) (middle) and iso-surface of the second invariant of the velocity gradient $\left(Q=1.0 \times 10^{6} \mathrm{~s}^{-2}\right)$ coloured by the axial velocity (bottom).

To explore the physical mechanisms related to the results shown in Figs. 8 and 9, the flow field results are examined from the present LES. Those with the single frequency forcing are presented in Fig. 10 for four typical cases, showing the heat release rate, temperature and the second invariant of the velocity gradient $(Q)$. The temperature flow fields show the same pattern as those for the heat release rate. For the case with $f_{1}=160 \mathrm{~Hz}$ and $A_{1}=0.1$, i.e. the primary forcing with relative small forcing amplitude, the fluctuation of the inlet velocity only changes the shear layers slightly and mushroom-shaped vortex can be barely observed. With increasing the forcing amplitude to $A_{1}=0.3\left(f_{1}=160\right.$ $\mathrm{Hz}$ ), the mushroom-shaped vortex is clearly visible in the flow fields which is the main factor for the non-linear heat release response for single frequency forcing as discussed previously [13, 22]. As to the harmonic forcing case with $f_{2}=320 \mathrm{~Hz}$ and $A_{2}=0.1$, there are two pairs of oscillations co-existing in the flow fields compared to the primary frequency forcing case. Also two pairs of mushroom-shaped vortex can be observed in the flow fields with relative large forcing amplitude of $A_{2}=0.3$. The flow structures indicated by the iso-surface of the second invariant of the velocity gradient $(Q)$ demonstrate that more structures appear with increasing the forcing amplitude for both the two 
frequencies. As to the forcing frequency, it seems that the primary forcing frequency at $f_{1}=160 \mathrm{~Hz}$ mainly affects the central recirculation and downstream regions, although effects on the side recirculation region along the vertical wall can be observed. For the harmonic forcing at $f_{2}=320 \mathrm{~Hz}$, it mainly affects the side recirculation region and vertical wall regions, especially when forcing with a relatively large forcing amplitude $A_{2}=0.3$ in which many structures emerge along the vertical wall in a complex pattern.

Similar processes are performed for four typical cases with two frequency forcing and the results are shown in Fig. 11. These four cases are the combinations of the cases with single frequency forcing shown in Fig. 10. Figure 11(a) presents the results of case $\mathrm{C} 11$, which can be seen as the combination of the two cases shown in Figs. 10(a) and 10(c). For the case C11, part of the forcing energy has been transferred from the primary forcing to the higher harmonic forcing as discussed previously. The flow structures demonstrate that more structures emerge near the vertical wall due to the structure interactions at the upper half region of the combustor. As to case C13, which are highly related to the results in Figs. 10(a) and 10(d), the flow is mainly governed by the harmonic forcing and that is the main reason for the similar flow structures of Fig. 11(b) and Fig. 10(d). Further to the case C31, accompanying the results in Figs. 10(b) and 10(c), it can be seen that the primary forcing dominates the flow evolution. However, the flow structures along the vertical wall become stronger in the upper half part of the combustor and they suppress the flow structures behind the central bluff body, which are supposed to contribute to the primary frequency forcing. Now for the case C33 in Fig. 11(d), which contains both strong primary forcing and harmonic forcing (see Figs. 10(b) and $10(d)$ ), a large flow structure distributed circumferentially can be clearly seen in the flow field. It probably results from the force balance between the movements of the central recirculation region and the more sensitive side recirculation region. It seems that the central recirculation region and side recirculation region have different responses to the acoustic forcing, regarding to the forcing frequency and forcing amplitude. The flame fronts generated between the two recirculation regions are thus affected by the balances between the two regions during the acoustic forcing.

\section{Conclusions}

Characterising and controlling the combustion instabilities encountered in gas turbine have been a long standing challenge. One of the key factors depends on the accurate representation of the non-linear flame response to acoustic waves under complex flow conditions. The present study has successfully performed a numerical study involving two frequencies in the acoustic waves which have been observed in experiments, but few numerical studies are conducted. This is achieved by using high-fidelity Large Eddy Simulations (LES), for a target case which is a bluff body stabilised, lean premixed flame combustor developed at Cambridge University.

The LES method which applies the open source CFD toolbox, OpenFOAM, is firstly validated for both unforced/forced reactive flow with single frequency forcing, for which some experimental data are available. Turbulent combustion is modelled using the Partial Stirred Reactor (PaSR) model with a reduced two-step reaction mechanism of ethylene/air. The results demonstrate that both the flow field and flame dynamics, as well as the unsteady heat release, are captured well by the present LES for the single frequency forcing cases. On the basis, LES calculations are then performed with acoustic forcing containing two frequencies. The primary forcing frequency is at $f_{1}=160 \mathrm{~Hz}$ and the harmonic forcing at $f_{2}=320 \mathrm{~Hz}$. Four forcing amplitudes at the primary frequency and two amplitudes for the harmonic frequency are considered varying from 0.1 up to 0.4 . When the second harmonic forcing is introduced to the primary forcing, the heat release responses are changed significantly. The amplitudes of heat release rate fluctuation responding at the primary frequency are reduced up to $70 \%$ less than those with single frequency forcing. For the phase results, the values are increased slightly when low level of harmonic forcing is introduced, while decreased significantly when relatively high level of harmonic forcing added. As to the heat release responses at the harmonic forcing under two frequency forcing conditions, the amplitudes are also generally decreased except one case in which both the forcing amplitudes at the two frequencies are small, i.e. $A_{1}=0.1$ and $A_{2}=0.1$. The phase results behave similarly to the amplitudes. Those results demonstrate that the combustion system becomes more stable when the second harmonic forcing is introduced. This thus lends strong support to design a method for controlling combustion instability by introducing a second frequency forcing [33]. The underlying physical mechanisms are examined. It is found that the heat release responses are highly related to the vortex structures in the flow field. For the specific cases studied here, the flow structures in the central recirculation region and side recirculation region have different responses to the primary and harmonic forcing with different amplitudes. This implies that the vortex flow structures can be excited or suppressed by introducing a second frequency forcing, and as such it changes the heat release rate 
responses. Finally the coupling between the acoustic waves and unsteady heat release could be decoupled and the combustion instability would be controlled.

This is the first work, to the authors' knowledge, which studies the two frequency effects for the Cambridge case using high-fidelity CFD method based on LES. This confirms that the open-source toolbox, OpenFOAM, can be used to study combustion instability problems numerically and good accuracy can be obtained.

\section{Acknowledgements}

Computation time using the CX1 HPC cluster at Imperial College is gratefully acknowledged.

\section{References}

[1] Swaminathan, N., Bray, K.N.C.. Turbulent Premixed Flames. Cambridge, UK: Cambridge University Press; 2014.

[2] Gövert, S., Mira, D., Kok, J., Vázquez, M., Houzeaux, G.. Turbulent combustion modelling of a confined premixed jet flame including heat loss effects using tabulated chemistry. Appl Energ 2015;156:804 - 815.

[3] Xiao, H., He, X., Duan, Q., Luo, X., Sun, J.. An investigation of premixed flame propagation in a closed combustion duct with a 90 bend. Appl Energ 2014;134:248 - 256.

[4] Poinsot, T., Veynante, D.. Theoretical and Numerical Combustion. second ed.; PA, USA: R.T. Edwards; 2005.

[5] Dowling, A.P., Stow, S.R.. Acoustic analysis of gas turbine combustors. J Propul Power 2003;19:751-763.

[6] Candel, S.. Combustion dynamics and control: Progress and challenges. Proc Combust Inst 2002;29(1):1-28.

[7] Lieuwen, T., Yang, V.. Combustion Instabilities in Gas Turbine Engines: Operational Experience, Fundamental Mechanisms, and Modeling. Reston, VA, USA: American Institute of Aeronautics and Astronautics, Inc.; 2005.

[8] Taamallah, S., Vogiatzaki, K., Alzahrani, F., Mokheimer, E., Habib, M., Ghoniem, A.. Fuel flexibility, stability and emissions in premixed hydrogen-rich gas turbine combustion: Technology, fundamentals, and numerical simulations. Applied Energy 2015;154:1020 - 1047.

[9] Stow, S.R., Dowling, A.P.. A time-domain network model for nonlinear thermoacoustic oscillations. J Eng Gas Turb Power 2009;131:031502.

[10] Palies, P., Durox, D., Schuller, T., Candel, S.. Nonlinear combustion instability analysis based on the flame describing function applied to turbulent premixed swirling flames. Combust Flame 2011;158:1980-1991.

[11] Crocco, L.. Aspects of combustion stability in liquid propellant rocket motors part i: Fundamentals. low frequency instability with monopropellants. J Am Rocket Soc 1951;21:163-178.

[12] Noiray, N., Durox, D., Schuller, T., Candel, S.. A unified framework for nonlinear combustion instability analysis based on the flame describing function. J Fluid Mech 2008;615:139-167.

[13] Balachandran, R., Ayoola, B.O., Kaminski, C.F., Dowling, A.P., Mastorakos, E.. Experimental investigation of the nonlinear response of turbulent premixed flames to imposed inlet velocity oscillations. Combust Flame 2005;143(12):37-55.

[14] Balachandran, R.. Experimental investigation of the response of turbulent premixed flames to acoustic oscillations. Ph.D. thesis; University of Cambridge; Cambridge, UK; 2005.

[15] Armitage, C.A., Balachandran, R., Mastorakos, E., Cant, R.S.. Investigation of the nonlinear response of turbulent premixed flames to imposed inlet velocity oscillations. Combust Flame 2006;146(3):419-436.

[16] Durox, D., Schuller, T., Noiray, N., Candel, S.. Experimental analysis of nonlinear flame transfer functions for different flame geometries. Proc Combust Inst 2009;32(1):1391-1398.

[17] Bellows, B.D., Bobba, M.K., Seitzman, J.M., Lieuwen, T.. Nonlinear flame transfer function characteristics in a swirl-stabilized combustor. J Eng Gas Turb Power 2007;129:954-961.

[18] Schimek, S., Moeck, J.P., Paschereit, C.O.. An experimental investigation of the nonlinear response of an atmospheric swirl-stabilized premixed flame. J Eng Gas Turb Power 2011;133:101502.

[19] Kim, D., Kim, K., Srinivasan, S., Lee, J.G., Quay, B.D., Santavicca, D.A.. Effect of flame structure on the flame transfer function in a premixed gas turbine combustor. J Eng Gas Turb Power 2010;132:021502.

[20] Ćosić, B., Terhaar, S., Moeck, J.P., Paschereit, C.O.. Response of a swirl-stabilized flame to simultaneous perturbations in equivalence ratio and velocity at high oscillation amplitudes. Combust Flame 2015;162(4):1046-1062.

[21] Lieuwen, T.. Modeling premixed combustion-acoustic wave interactions: A review. J Propul Power 2003;19:765-781.

[22] Han, X., Morgans, A.S.. Simulation of the flame describing function of a turbulent premixed flame using an open-source LES solver. Combust Flame 2015;162(5):1778-1792.

[23] Han, X., Li, J., Morgans, A.S.. Prediction of combustion instability limit cycle oscillations by combining flame describing function simulations with a thermoacoustic network model. Combust Flame 2015;162(10):3632 - 3647.

[24] Krediet, H.J., Beck, C.H., Krebs, W., Kok, J.B.W.. Saturation mechanism of the heat release response of a premixed swirl flame using LES. Proc Combust Inst 2013;34(1):1223 - 1230.

[25] Jones, W., Marquis, A., Wang, F.. Large eddy simulation of a premixed propane turbulent bluff body flame using the eulerian stochastic field method. Fuel 2015;140:514 - 525.

[26] Zhou, L., Lu, Z., Ren, Z., Lu, T., Luo, K.H.. Large eddy simulation of an n-heptane spray flame with dynamic adaptive chemistry under different oxygen concentrations. SAE Int J Engines 2015;8:447 - 454.

[27] Wu, Y., Carlsson, C., Szasz, R., Peng, L., Fuchs, L., Bai, X.S.. Effect of geometrical contraction on vortex breakdown of swirling turbulent flow in a model combustor. Fuel 2016;170:210 - 225. 
[28] Balachandran, R., Dowling, A.P., Mastorakos, E.. Non-linear response of turbulent premixed flames to imposed inlet velocity oscillations of two frequencies. Flow Turbul Combust 2008;80:455 - 487.

[29] Joos, F., Vortmeyer, D.. Self-excited oscillations in combustion chambers with premixed flames and several frequencies. Combust Flame $1986 ; 65(3): 253-262$.

[30] Paschereit, C.O., Gutmark, E., Weisenstein, W.. Excitation of thermoacoustic instabilities by interaction of acoustics and unstable swirling flow. AIAA J 2000;38:1025 - 1034

[31] Lieuwen, T.C.. Unsteady combustor physics. New York: Cambridge University Press; 2012.

[32] Emerson, B., Lundrigan, J., O’Connor, J., Noble, D., Lieuwen, T.. Convective and absolute instabilities in reacting bluff body wakes. In: Proc ASME Turbo Expo 2011. 2011,GT2011-45330.

[33] Ćosić, B., Bobusch, B.C., Moeck, J.P., Paschereit, C.O.. Open-loop control of combustion instabilities and the role of the flame response to two-frequency forcing. J Eng Gas Turb Power 2012;134:061502.

[34] Swaminathan, N., Xu, G., Dowling, A.P., Balachandran, R.. Heat release rate correlation and combustion noise in premixed flames. J Fluid Mech 2011;681:80-115.

[35] Gong, C., Jangi, M., Bai, X.S.. Large eddy simulation of n-dodecane spray combustion in a high pressure combustion vessel. Appl Energ $2014 ; 136: 373-381$.

[36] Selle, L., Lartigue, G., Poinsot, T., Koch, R., Schildmacher, K.U., Krebs, W., et al. Compressible large eddy simulation of turbulent combustion in complex geometry on unstructured meshes. Combust Flame 2004;137(4):489-505.

[37] Smagorinsky, J.. General circulation experiments with the primitive equations: I the basic experiment. Mon Weather Rev 1963;91:99-164.

[38] Pope, S.B.. Turbulent Flows. Cambridge University Press; 2000.

[39] Fureby, C.. A comparative study of flamelet and finite rate chemistry LES for a swirl stabilized flame. J Eng Gas Turb Power 2012;134:041503.

[40] Afarin, Y., Tabejamaat, S.. The effect of fuel inlet turbulence intensity on h2/ch4 flame structure of mild combustion using the LES method. Combust Theor Model 2013;17(3):383-410.

[41] Chomiak, J., Karlsson, A.. Flame liftoff in diesel sprays. Symp (Int) Combustion 1996;26(2):2557-2564.

[42] Cuenot, B., Riber, E., Franzelli, B.. Towards the prediction of soot in aero-engine combustors with large eddy simulation. Tech. Rep.; Center for Turbulence Research, Proceedings of the Summer Program 2014; 2014.

[43] Sabelnikov, V., Fureby, C.. LES combustion modeling for high re flames using a multi-phase analogy. Combust Flame 2013;160(1):83-96.

[44] Fureby, C., Nordin-Bates, K., Petterson, K., Bresson, A., Sabelnikov, V.. A computational study of supersonic combustion in strut injector and hypermixer flow fields. Proc Combust Inst 2015;35(2):2127-2135.

[45] Tay-Wo-Chong, L., Polifke, W.. Large eddy simulation-based study of the influence of thermal boundary condition and combustor confinement on premix flame transfer functions. J Eng Gas Turb Power 2013;135(1):021502.

[46] Mejia, D., Selle, L., Bazile, R., Poinsot, T.. Wall-temperature impact effects on flame response to acoustic oscillations. Proc Combust Inst 2015;35(3):3201-3208.

[47] Bauerheim, M., Staffelbach, G., Worth, N., Dawson, J., Gicquel, L., Poinsot, T.. Sensitivity of LES-based harmonic flame response model for turbulent swirled flames and impact on the stability of azimuthal modes. Proc Combust Inst 2015;35(3):3355-3363. 